\title{
Phase transitional behaviors of bent-cored liquid crystal in electric field
}

\author{
Peng Huan-Gao ${ }^{1}$, Zicong Zhou ${ }^{3}$, Holger Merlitz ${ }^{1}$, Wu Chen-Xu ${ }^{1,2}$ \\ 1 Institute of Softmatter and Biometrics, and ${ }^{2}$ Collaborative Innovation Center of \\ Chemistry for Energy Materials, Xiamen University, Xiamen 361005, PR China \\ and ${ }^{3}$ Department of Physics, Tamkang University, Taiwan
}

\begin{abstract}
Monte Carlo(MC) simulations based on lattice model were performed to study the phase diagram(anisotropy,uniaxiality and biaxiality) of liquid crystals formed by bent-cored molecules with a strong transverse dipole moment deviating from their angular bisector. It is shown that the asymmetric strong dipolar interaction enhances biaxiality slightly but encourages uniaxiality greatly and as a result suppresses the system's isotropic order, which is different from a system free from external field in that dipole moment increases biaxiality by suppressing the uniaxial and the isotropic orders simultaneously. It is also found that an external electric field encourages the biaxiality slightly but considerably enhances the uniaxiality of bent-cored liquid crystal.
\end{abstract}

PACS numbers: PACS: $61.30 . \mathrm{Cz}, 61.30 . \mathrm{Gd}, 64.70 . \mathrm{Md}$

\section{INTRODUCTION}

As a kind of functional materials, liquid crystal has been widely used in electro-optic display devices for many years. Recently, much attention has been paid to biaxial nematic liquid crystals for their potential applications in fast-switching display devices. ${ }^{[1]}$ In 1970 s, Freiser ${ }^{[2]}$ used a molecular field theory to exhibit that molecules deviating from cylindrical symmetry can construct not only a uniaxial nematic phase but also a biaxial one. Since then, stable biaxial nematic phase has been observed in experiments in lyotropic system in $1980^{[3]}$ and in polymeric system in $2004^{[4]}$ respectively, but not in thermotropic system ${ }^{[5,6]}$ in spite of the positive support by computer simulations. ${ }^{[7-10]}$ Meanwhile, besides Freiser's contribution, many theories such as mean field theory, ${ }^{[11-13]}$ bifurcation analysis, ${ }^{[14]}$ density functional theory, ${ }^{[15]}$ statistical theory ${ }^{[16,17]}$ etc, also supported the existence of biaxial nematic phase. In 2004, Madsen et al. ${ }^{[18]}$ and Acharya et al. ${ }^{[19]}$ proclaimed that bent-cored molecules may form an elusive biaxial nematic phase, an effect studied experimentally in recent years. ${ }^{[20,21]}$ As a result, a typical phase diagram, which consists of four phases: two uniaxial nematic $\left(N_{U}\right)$ phases, a biaxial nematic $\left(N_{B}\right)$ phase and an isotropic (I) phase, has been proved by theoretical studies using models for bent-cored molecules ${ }^{[22-24]}$ and computer simulations ${ }^{[25,26]}$ respectively. It is shown that such a system formed by bent-cored liquid crystal molecules undergoes a transition directly from isotropic phase to biaxial nematic phase when Landau point appears at a single tetrahedral angle of $109.47^{\circ}$. However, while taking a strong transverse electric dipole moment into account, in Madsen's theory, a much larger tetrahedral angle is predicted for Landau point to appear. In order to explore these kinds of transitional behaviors, Osipov et al. ${ }^{[2]}$ used molecular theory and Bates ${ }^{[28]}$ performed a series of Monte Carlo (MC) simulations based on a lattice model to study the system with a transverse dipole bisecting their bend angle. They showed that the direct phase transition from isotropic nematic phase to biaxial nematic occurs in a range of angles rather than a single one, leading to a Landau line rather than a Landau point, and the transition becomes first-order. What if the electric dipole moment does not fall into the direction of the bent-cored angular bisector? To address this question, Ye ${ }^{[29]}$ performed a series of MC simulations for liquid crystals formed by asymmetric bent-cored molecules with a strong transverse dipole moment deviating from their angular bisector. It was found that the asymmetry due to the orientation of dipole moment suppresses uniaxiality in the small-bend angle regime, but encourages biaxial phase regime in the phase diagram. As liquid crystal is very sensitive to small disturbance from its surrounding, it is practically important to study the influence of electric and magnetic field ${ }^{[30]}$ in liquid crystals formed by bent-cored molecules.

In this paper, we carried out a series of MC simulations to investigate the phase transitional behaviors of the bent-cored molecules in temperature domain in an electric field. The paper is organized as follows. The simulation model and theory are introduced in Section 2. In Section 3, the phase transitional behaviors of liquid crystals formed by bent-cored molecules with and without an electric field are analyzed and discussed. Our conclusion is presented in Section 4.

\section{SIMULATION MODEL AND MEAN-FIELD THEORY}

\section{II.1. SIMULATION MODEL}

Here we consider a group of biaxial bent-cored molecules. ${ }^{[29]}$ Each molecule is assumed to have two identical rods (rod 1 and rod 2) jointed at a fixed bend angle $\theta$ and possess a dipole moment $\boldsymbol{\mu}$ shifting an angle from its bent-cored angular bisector, as shown in FIG.1. For simplicity, one rod is located in the $y z$ plane of the molecular frame $\{\boldsymbol{x}, \boldsymbol{y}, \boldsymbol{z}\}$ with $y$ axis bisecting its bend angle. The deviation angle of the molecular dipole moment from $y$ axis is denoted by $\varphi$, and an electric field is applied to the system parallel to $Z$ axis in laboratory 


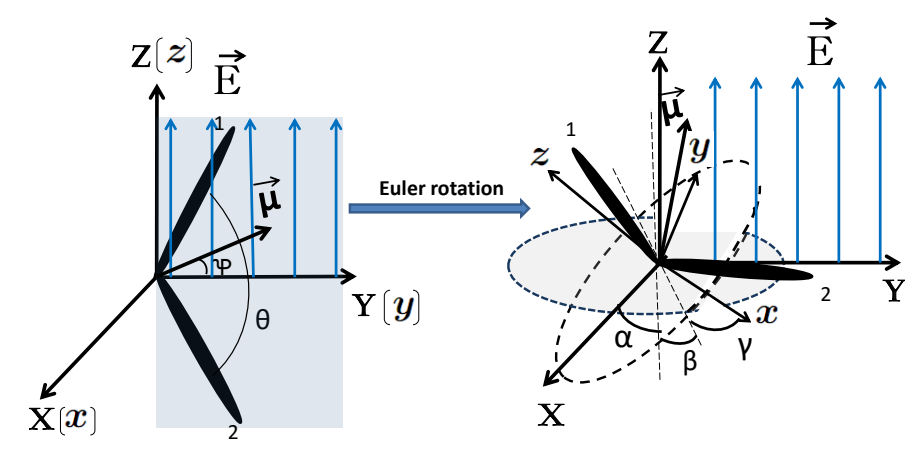

FIG. 1. Schematic of a bent-cored molecule with an electric dipole moment $\boldsymbol{\mu}$ in an electric field.

frame.

The interaction between the dipole and the electric field results in an energy

$$
\delta E=-\boldsymbol{\mu} \cdot \boldsymbol{E}
$$

where $\boldsymbol{\mu}$ and $\boldsymbol{E}$ are vectors of dipole moment and electric field, respectively. We set $\boldsymbol{\mu}=\kappa \boldsymbol{u}$ and $\boldsymbol{E}=\lambda(0,0,1)$, where $\boldsymbol{u}$ is unit vector of the dipole moment, and parameters $\kappa$ and $\lambda$ represent the reduced strengths of dipole moment and electric field, respectively. The molecular frame can be connected to laboratory frame via operations $R_{z}(-\alpha) R_{y}(\beta) R_{z}(-\gamma)$, corresponding to a rotation matrix given by

$$
\begin{aligned}
& \Omega(\alpha, \beta, \gamma)= \\
& \left(\begin{array}{ccc}
a_{11} & a_{12} & \cos \alpha \sin \beta \\
a_{21} & a_{22} & \sin \alpha \sin \beta \\
-\sin \beta \cos \gamma & \sin \beta \sin \gamma & \cos \beta
\end{array}\right),
\end{aligned}
$$

with

$$
\begin{aligned}
& a_{11}=\cos \alpha \cos \beta \cos \gamma-\sin \alpha \sin \gamma \\
& a_{12}=-\cos \alpha \cos \beta \sin \gamma-\sin \alpha \cos \gamma \\
& a_{21}=\sin \alpha \cos \beta \cos \gamma+\cos \alpha \sin \gamma \\
& a_{22}=-\sin \alpha \cos \beta \sin \gamma+\cos \alpha \cos \gamma
\end{aligned}
$$

where $\alpha, \beta$ and $\gamma$ are three Euler angles.

The unit vectors along the two rods $\boldsymbol{u}_{1}$ and $\boldsymbol{u}_{2}$, and the unit vector of the dipole moment $\boldsymbol{u}$ of each molecule, in laboratory frame, can then be written in terms of Euler angles as

$$
\begin{gathered}
\boldsymbol{u}_{1}=\Omega(\alpha, \beta, \gamma)\left(\begin{array}{c}
0 \\
\cos \theta / 2 \\
\sin \theta / 2
\end{array}\right) \\
\boldsymbol{u}_{2}=\Omega(\alpha, \beta, \gamma)\left(\begin{array}{c}
0 \\
\cos \theta / 2 \\
-\sin \theta / 2
\end{array}\right) \\
\boldsymbol{u}=\Omega(\alpha, \beta, \gamma)\left(\begin{array}{c}
0 \\
\cos \varphi \\
\sin \varphi
\end{array}\right) .
\end{gathered}
$$

Given Eq. (1) and Eq. (5), the energy between the external field and the bent-cored molecule is

$$
\begin{aligned}
\delta E & =-\kappa \lambda(0,0,1) \Omega(\alpha, \beta, \gamma)\left(\begin{array}{c}
0 \\
\cos \varphi \\
\sin \varphi
\end{array}\right) \\
& =-\kappa \lambda(\cos \varphi \sin \beta \sin \gamma+\sin \varphi \cos \beta) .
\end{aligned}
$$

Similar to the Lebwohl-Lasher model, ${ }^{[31]}$ we assign the system in a cubic lattice and assume that each molecule interacts only with its six nearest neighbors, leading to an interaction energy between a molecule and its nearest neighbors

$$
U_{i}=\sum_{j=1}^{6}\left(U_{i j}^{a n i s}+U_{i j}^{d d}\right)
$$

where $U_{i j}^{\text {anis }}$ is an anisotropic potential between molecules $i$ and $j$ given by [29]

$$
U_{i j}^{\text {anis }}=-\sum_{m=1,2} \sum_{n=1,2} \epsilon P_{2}\left(\cos \beta_{i_{m} j_{n}}\right) .
$$

Here the indices $m$ and $n$ run over the two rods in molecules $i$ and $j$ respectively, $\epsilon$ is the energy parameter which is supposed to be a positive constant when the molecules $i$ and $j$ are the nearest neighbors or zero otherwise, $\beta_{i_{m} j_{n}}$ is the angle between $\operatorname{rod} m$ in molecule $i$ and $\operatorname{rod} n$ in molecule $j$, and $P_{2}(\cos \beta)=3\left(\cos ^{2} \beta-1\right) / 2$ is the second Legendre polynomial. The general form of the dipole-dipole interaction, $U_{i j}^{d d}$, is

$$
U_{i j}^{d d}=\frac{\mu^{2}}{4 \pi \epsilon_{0} r_{i j}^{3}}\left[\left(\boldsymbol{u}_{i} \cdot \boldsymbol{u}_{j}\right)-3\left(\boldsymbol{u}_{i} \cdot \hat{\boldsymbol{r}}_{i j}\right)\left(\boldsymbol{u}_{j} \cdot \hat{\boldsymbol{r}}_{i j}\right)\right],
$$

where $\boldsymbol{u}_{i}$ is the unit vector of molecular permanent polarization, $\hat{\boldsymbol{r}}_{i j}$ is the unit vector of the intermolecular centerof-mass separation $r_{i j}$ between molecules $i$ and $j$, and $\epsilon_{0}$ is the permittivity in vacuum. In the lattice model, the orientation angles are discretized and the orientational order of the system with anisotropic interactions is determined by the intermolecular orientation, and thus is pinned along a lattice axis. ${ }^{[28]}$ Instead of using Eq. (9), we use a much simpler energy which merely depends on the relative orientation of neighboring dipolar pairs

$$
U_{i j}^{d d}=\frac{\mu^{2}}{4 \pi \epsilon_{0} r_{i j}^{3}} \cos \eta
$$


where $\cos \eta=\boldsymbol{u}_{i} \cdot \boldsymbol{u}_{j}$.

The total energy of each molecule in our simulation, therefore, is

$$
E_{i}=\sum_{j=1}^{6}\left(U_{i j}^{a n i s}+U_{i j}^{d d}\right)+\delta E_{i} .
$$

There are five parameters in the total interaction energy: (1) $\theta$ : the bend angle between $\operatorname{rod} 1$ and $\operatorname{rod} 2 ;(2) \varphi$ : the deviation angle of molecular permanent polarization from the bisector; (3) $\kappa=\mu^{2} /\left(4 \pi \epsilon_{0} r_{i j}^{3} \epsilon\right)$ : the relative strength of dipole moment; (4) $\lambda$ : the strength of the applied electric field and is scaled in $\kappa$; (5) $T^{*}=k_{B} T / \epsilon$ : the dimensionless temperature, where $k_{B}$ is the Boltzmann constant.

We performed Metropolis MC simulations [32] to explore the phase diagram of the system in vacuum, with and without an external electric field, on a cubic lattice of size $10 \times 10 \times 10$ with periodic boundary condition, which is large enough, as we did and checked in our previous paper. ${ }^{[29]}$ Each MC step was made by selecting a site randomly and calculating the energy of this configuration $\left(U_{1}\right)$, followed by a random change of orientation and calculate the energy of this new configuration $\left(U_{2}\right)$. Equilibration runs are typically 100000 sweeps with each sweep consist of $N=10^{3} \mathrm{MC}$ steps, followed by a thermal average run of 10000 sweeps. Longer equilibration runs were performed in the vicinity of phase transitions.

The MC simulation researches in vacuum based on the model $(\lambda=0)$ have been done by Ye et. al. ${ }^{[2]}$ The results show that strong dipolar interaction enhances system biaxiality considerably in the whole region while the deviation of the dipole moment from its angular bisector of bent-cored molecules suppresses uniaxiality only in the small bend angle region.

The order parameters for the arm axes were found to possess the most useful information on phase behaviors in the previous study, ${ }^{[25]}$ which were also applied to our dipolar model in electric field. The phase transitional behaviors of biaxial bent-cored liquid crystal were investigated in terms of dimensionless temperature $T^{*}$, bend angle $\theta$, relative strength of dipole moment $\kappa$, dipole moment deviation angle $\varphi$ and the strength of electric field $\lambda$.

\section{II.2. MEAN-FIELD THEORY}

Different from that in the uniaxial nematic phase, the orientational order of the rigid biaxial molecule in a biaxial phase can be characterized by a Cartesian super matrix $S$ with elements ${ }^{[25]}$

$$
S_{a b}^{A B}=\left\langle\frac{3 l_{a A} l_{b B}-\delta_{a b} \delta_{A B}}{2}\right\rangle,
$$

where the parameters $a$ and $b$ denote the molecular frame, and $A, B$ denote the laboratory axes $(X, Y, Z)$ respectively. $l_{a A}$ is the direction cosine between the axis $a$ and the axis $A, \delta_{a b}$ is Kronecker delta function, and the angular bracket represents the ensemble average. Here it should be noted that as what we are interested in is the biaxiality of the system, we did not include the polar order, though exists, into our discussion.

During simulation, as the principal axes of the phase (mean orientation of molecules) change from configuration to configuration are unknown, we use the procedure described by Vieillard-Baron, ${ }^{[33]}$ by defining a $Q$ tensor for axes set in the molecule, in analogue to a diagonal matrix of $S^{[25]}$

$$
Q_{a a}^{A B}=\left\langle\frac{3 l_{a A} l_{a B}-\delta_{A B}}{2}\right\rangle .
$$

Diagonalizing the matrix we obtain three eigenvectors or directors. The three eigenvalues, $Q_{a a}^{A A}(A=X, Y$, or $Z)$, are the order parameters of axis $a$ with respect to the directors. We refer the largest eigenvalue as $Q_{a a}^{Z Z}$. Theoretically, the phase of system is determined by the three eigenvalues: $Q_{a a}^{X X} \neq Q_{a a}^{Y Y} \neq Q_{a a}^{Z Z} \neq 0$ gives a biaxial phase, $Q_{a a}^{X X}=Q_{a a}^{Y Y}=-Q_{a a}^{Z Z} / 2$ corresponds to a uniaxial phase, and $Q_{a a}^{X X}=Q_{a a}^{Y Y}=Q_{a a}^{Z Z}=0$ defines an isotropic phase. ${ }^{[29]}$ For convenience, we may let $Q_{a a}^{X X}=$ $(-S-\eta) / 2, Q_{a a}^{Y Y}=(-S+\eta) / 2$ and $Q_{a a}^{Y Y}=S$. According to the symmetry, phenomenologically we may write an expansion of the free energy in terms of the invariants of the order parameter tensor, $I_{n}=\operatorname{Tr} Q^{n}$, with $n=$ $1,2,3 \cdots$. Due to the symmetry of $Q$, all these invariants can be written as polynomials depending on two basic invariants, given by ${ }^{[34]}$

$$
\begin{aligned}
& I_{2}=\operatorname{Tr} Q^{2}=\frac{1}{2}\left(3 S^{2}+\eta^{2}\right) \\
& I_{3}=\operatorname{Tr} Q^{3}=\frac{3}{4} S\left(S^{2}-\eta^{2}\right) .
\end{aligned}
$$

Given these, the Landau-de Gennes free energy can be written as

$f=f_{0}-h S+\frac{A}{2} I_{2}+\frac{B}{3} I_{3}+\frac{C}{4} I_{2}^{2}+\frac{1}{5} D I_{2} I_{3}+\frac{E}{6} I_{2}^{3}+E^{\prime} I_{3}^{2}+\cdots$

where $h$ is the external field applied to the system, $A$ is related to temperature and $B$ is related to molecular biaxiality. The above phenomenological expansion gives a Landau multicritical point for $A=B=0$ when $h=0$. Nascimento et al. used standard statistical mechanics to obtain a global phase diagram, including isotropic order, uniaxial and biaxial phases, a reentrant ordered region, and multicritical point. ${ }^{[34]}$ To plot the figure of $I-N_{U}$ and $N_{U}-N_{B}$ phase transition, for convenience, we let $E=D=0$ in the real calculation.

In equilibrium state when no external field is applied $(h=0)$, the minimization of free energy Eq. (16) leads to $[35]$

$$
A=-\frac{3}{2} C S^{2}, B=-\frac{9}{2} E^{\prime} S^{3},
$$


which gives the critical line for $N_{U}-N_{B}$ by eliminating $S$. To get the phase transition condition from isotropic to uniaxial, we let $\eta=0$ and obtain

$$
A=\frac{3}{4} C S^{2}+\frac{9}{4} E^{\prime} S^{4}, B=-\frac{9}{2} C S-9 E^{\prime} S^{3},
$$

which defines the critical line for $I-N_{U}$.

When an external field is applied $(h \neq 0)$, using similar approach, we have

$$
A=\frac{9 h}{4 S}-\frac{3}{2} C S^{2}, B=\frac{4 h}{9 S^{2}}-\frac{9}{2} E^{\prime} S^{3}
$$

and

$$
A=\frac{3}{4} C S^{2}+\frac{9}{4} E^{\prime} S^{4}+\frac{8 h}{3 S}, B=-\frac{9}{2} C S-9 E^{\prime} S^{3}-\frac{4 h}{S^{2}}
$$

for the critical lines of $N_{U}-N_{B}$ and $I-N_{U}$ respectively. It is found from Fig. (2) that the application of external field encourages biaxiality slightly but enlarges the regime of uniaxiality considerably.

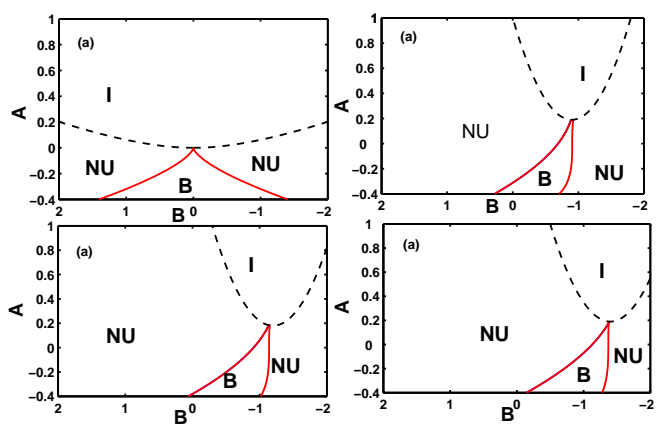

FIG. 2. The phase diagrams of liquid crystal formed by permanently polarized bent-cored molecules in terms of $A$ and $B$. $A$ is a measure for temperature, and $B$ represents the degree of flatness of molecules. $C=0.5, E^{\prime}=0.8$, and external fields $(\mathrm{a}) h=0 ;(\mathrm{b}) h=0.06 ;(\mathrm{c}) h=0.08 ;(\mathrm{d}) h=0.1$.

\section{SIMULATION RESULTS}

Figure 3 shows the phase diagram in terms of bend angle $\theta$ and temperature $T^{*}$ for different deviated dipole moments $(\kappa=0,0.5,1.5,2)$. When $\kappa=0$, the uniaxial nematic phases were separated into two regions by a Landau point $\theta=109.47^{\circ}\left(\cos ^{-1} \theta=-1 / 3\right)$, which is in good agreement with the prediction by molecular field theory. ${ }^{[23]}$ However, once the dipole-dipole interaction is taken into account, such as $\kappa=0.5$, one sees a slight increase of phase transition temperature for $N_{U}-N_{B}$ (which is second-order ${ }^{[34]}$ ), an apparent increase in small $\theta$ region for $I-N_{U}$ (first-order), and a larger Landau point of $\theta=120^{\circ}$ enabling an $I-N_{B}$ transition. Though the system has no isotropic symmetry due to the application of external field, we still use notation $I$ for isotropic
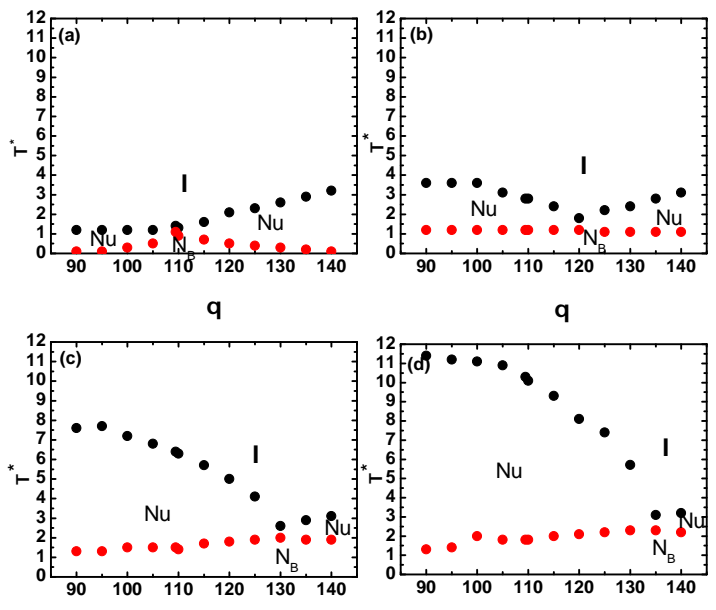

$\theta$

FIG. 3. The phase diagrams of liquid crystal formed by permanently polarized bent-cored molecules in terms of the dimensionless temperature $T^{*}\left(T^{*}=k_{B} T / \epsilon\right)$ and the bend angle $\theta$ between rod 1 and rod 2 for different polarization strength $\left(\varphi=5^{\circ}\right.$ and $\lambda=2$ ): (a) $\kappa=0$; (b) $\kappa=0.5$; (c) $\kappa=1 ;$ (d) $\kappa=1.5$.

phase, here referring to zero orientational order. From Fig. 3, it is also shown that under the application of an external field, as the deviated dipole moment increases, the biaxiality of the system is slightly encouraged, while the isotropic phase is greatly suppressed. The uniaxiality of the system, however, shows an unbalance behavior in small and large bend angle regime. It is favored for small bend angle while disfavored if it is larger than Landau point.
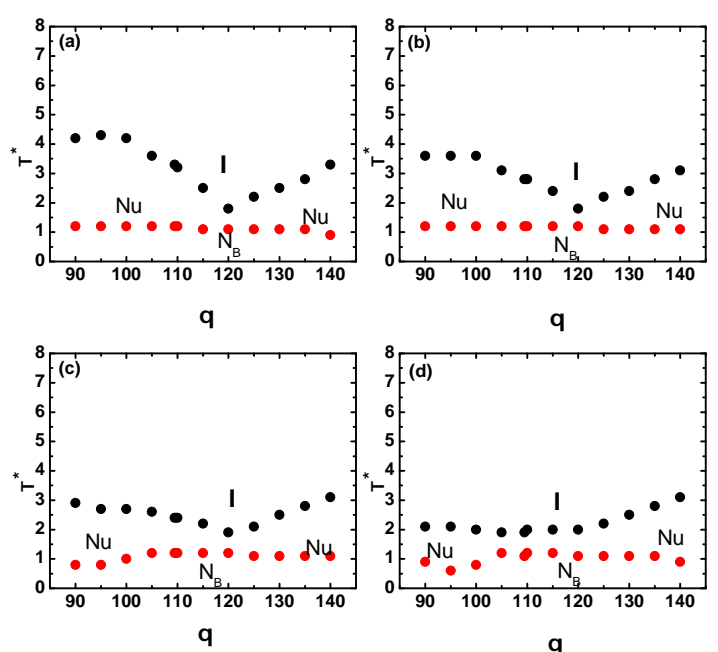

FIG. 4. The phase diagrams of liquid crystal formed by bentcored molecules in terms of the dimensionless temperature $T^{*}\left(T^{*}=k_{B} T / \epsilon\right)$ and the bend angle $\theta$ between rod 1 and rod 2 , for different deviation angles $(\kappa=0.5$ and $\lambda=2)$ : (a) $\varphi=0^{\circ}$; (b) $\varphi=5^{\circ}$; (c) $\varphi=10^{\circ}$; (d) $\varphi=15^{\circ}$. 
We also study the effect of bend angle on phase diagram for different deviation angles of dipole moment from molecular bisector, as shown in Fig. 4. It is found that under an external field, an increasing deviation , an indication of the asymmetric permanent polarization, favors the isotropic orientation of the system but suppresses its uniaxiality, especially in low bend angle region. The biaxility of the system does not change much, but the Landau point disappears, indicating that large deviation denies the direct transition from isotropic phase to biaxial phase.
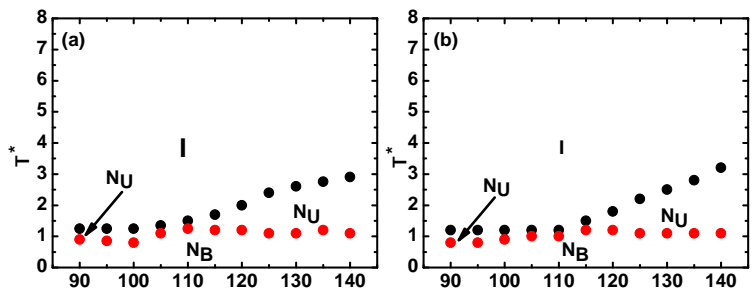

$\theta$
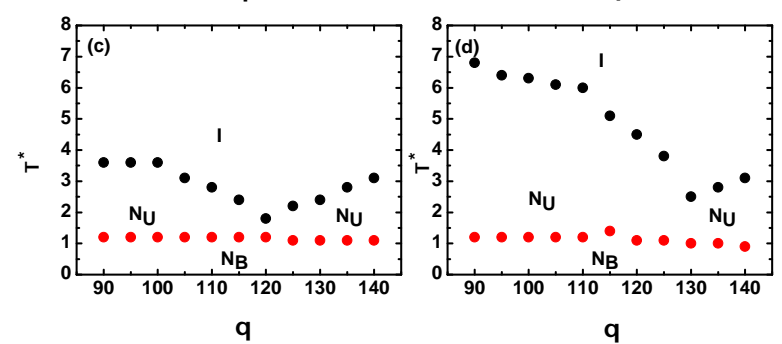

FIG. 5. The phase diagrams of liquid crystal formed by bentcored molecules in terms of the dimensionless temperature $T^{*}\left(T^{*}=k_{B} T / \epsilon\right)$ and the bend angle $\theta$ between $\operatorname{rod} 1$ and rod 2 , with $\kappa=0.5$ and $\varphi=5^{\circ}$, for different field strengths: (a) $\lambda=0$; (b) $\lambda=1$; (c) $\lambda=2$; (d) $\lambda=3$.

It is interesting to investigate the effect of external field on phase diagram. When a weak electric field is applied, such as $\lambda=1$ in Fig. 5, the Landau point does not change $\left(\theta=109.47^{\circ}\right)$. However, as the external field increases further, the uniaxiality of the system is enhanced substantially by squeezing the isotropic regime, especially in small bend angle regime, as shown in Fig. 5(c)-(d). This agrees with the theoretical estimation expected by mean theory(Fig. 2). There seems to be no sensitive correlation between external field and biaxiality.

Finally, we also studied the dependence of phase diagram on bend angle, as shown in Fig. 6. It is found that when $\theta=95^{\circ}$, there exists both $I-N_{U}$ transition and $N_{U}-N_{B}$ transition but no $I-N_{B}$ transition. In contrast, at larger $\theta$, Landau points appears, leading to the possibility of $I-N_{B}$ transition. Overall, bend angle plays a less important role in the phase structure of this system.
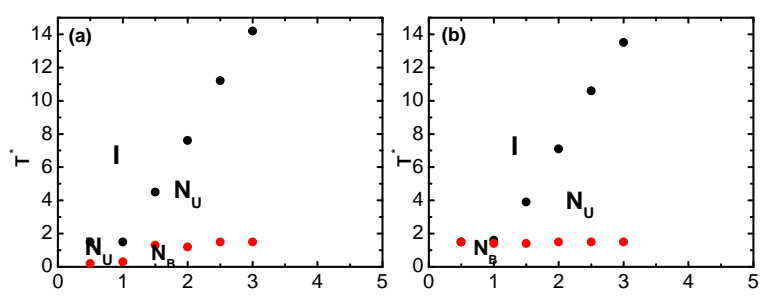

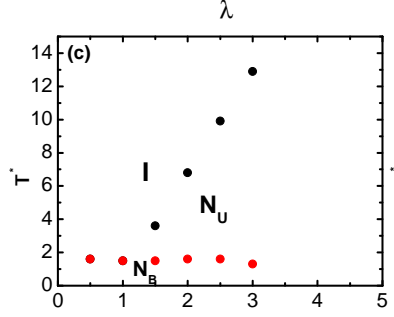

$\lambda$

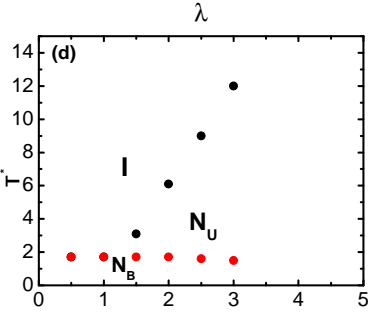

$\lambda$
FIG. 6. The phase diagrams of liquid crystal formed by bentcore molecules, with $\kappa=1$ and $\varphi=5^{\circ}$, in terms of dimensionless temperature $T^{*}\left(T^{*}=k_{B} T / \epsilon\right)$ and applied electric field $\lambda$ for four different bent angles: (a) $\theta=95^{\circ}$; (b) $\theta=105^{\circ}$; (c) $\theta=110^{\circ} ;$ (d) $\theta=115^{\circ}$.

\section{CONCLUSION}

In summary, MC simulations were performed to study the phase diagram of on a liquid crystal system formed by bent-cored molecules with a dipole moment deviating from their molecular angular bisector under an external electric field. A simple mean-field theory is presented to explain the phase diagram structure. It is shown that strong dipolar interaction enhances biaxiality slightly but encourages uniaxiality greatly and as a result suppresses the system's isotropic order. This is different from a system free from external field in which dipole moment increases biaxiality by suppressing the uniaxial and the isotropic orders simultaneously. The deviation of dipole moment from the angular bisector of molecules suppresses the system's uniaxiality by enhancing its isotropic order in small bend-angle regime, contrasting to the enhancement of biaxiality by suppressing uniaxiality in a system free from external field. This indicates that an electric field, when applied to a liquid crystal system formed by bent-cored molecules with a dipole moment deviating from their molecular angular bisector, in association with molecular dipole moment, encourages biaxiality slightly but enlarges the regime of uniaxiality greatly, which agrees with what is expected by mean-field theory.

\section{ACKNOWLEDGMENTS}

This work was supported by the National Science Foundation of China under Grant Nos. 11374243 and 


\section{REFERENCE}

[1] Kumar S 2009 U. S. Patent 7604850

[2] Freiser M J 1970 Phys. Rev. Lett. 241041

[3] Yu L J and Saupe A 1980 Phys. Rev. Lett. 451000

[4] Severing K and Saalwachter K 2004 Phys. Rev. Lett. 92 125501

[5] Shenouda I G, Shi Y and Neubert M E 1994 Mol. Cryst. Liq. Cryst. 257209

[6] Hughes J R, Kothe G, Luckhurst G R et al 1997 J. Chem. Phys. 1079252

[7] Luckhurst G R and Romano S 1980 Mol. Phys. 40129

[8] Allen M P 1990 Liq. Cryst 8499

[9] Biscarini F, Chiccoli C, Pasini P, Semeria F and Zannoni C 1995 Phys. Rev. Lett. 751803

[10] Camp P J and Allen M P 1997 J. Chem. Phys. 1066681

[11] Straley J P 1974 Phys. Rev. A 101881

[12] Luckhurst G R, Zannoni C, Nordio P L and Segre U 1975 Mol. Phys. 301345

[13] Remler D K and Haymet A D J 1986 J. Phys. Chem. 90 5426

[14] Matteis G D, Romano S and Virga E G 2005 Phys. Rev. E 72041706

[15] Holyst R and Poniewierski A 1990 Mol. Phys. 69193

[16] Zhang Z D, Zhang D X and Sun Y B 2000 Chin. Phys. Lett 17749

[17] Zhang Z D, Zhang Y J and Sun Z L 2006 Chin. Phys. Lett 113025
[18] Madsenm L A, Dingemans T J, Nakata M and Samulski E T 2004 Phys. Rev. Lett. 92145505

[19] Acharya B R, Primak A and Kumar S 2004 Phys. Rev. Lett. 92145506

[20] Xiang Y, Goodby J W, Gortz V and Gleeson H F 2009 Appl. Phys. Lett. 19193507

[21] Kaur S, Belaissaoui A, Goodby J W, Gortz V and Gleeson H F 2011 Phys. Rev. E. 83041704

[22] Teixeira P I C, Masters A J and Mulder B 1998 Mol. Cryst. Liq. Cryst. 323167

[23] Luckhurst G R 2001 Thin Solid Films 39340

[24] Grzybowski P and Longa L 2011 Phys. Rev. Lett. 107 027802 fig1fig5

[25] Bates M A and Luckhurst G R 2005 Phys. Rev. E. 72 051702

[26] Bates M A 2006 Phys. Rev. E. 74061702

[27] Osipov M A and Pajak G 2012 J. Phys.: Condensed Matter 24142201

[28] Bates M A 2007 Chem. Phys. Lett. 437189

[29] YE Xiao-Fang J 2013 Chin.Phys.Lett. 30106103

[30] Prabir K. Mukherjee,Muklesur Rahman Chemical Physics. 4232013 178-181

[31] Lebwohl P A and Lasher G 1972 Phys. Rev. A 6426

[32] Binder K 1984 Applications of the Monte Carlo Method in Statistical Physics (Berlin/Heidelberg/New York/Tokyo:Springer-Verlag)

[33] Vieillard-baron J 1972 J. Chem. Phys. 564729

[34] Nascimento E S, Henriques E F, Vieira A P, and Salinas 2015 Phys. Rev. E 92062503

[35] Gramsbergen E F, Longa L and de jeu W H 1986 Phys. Rep. 135195 
Graphical abstract:
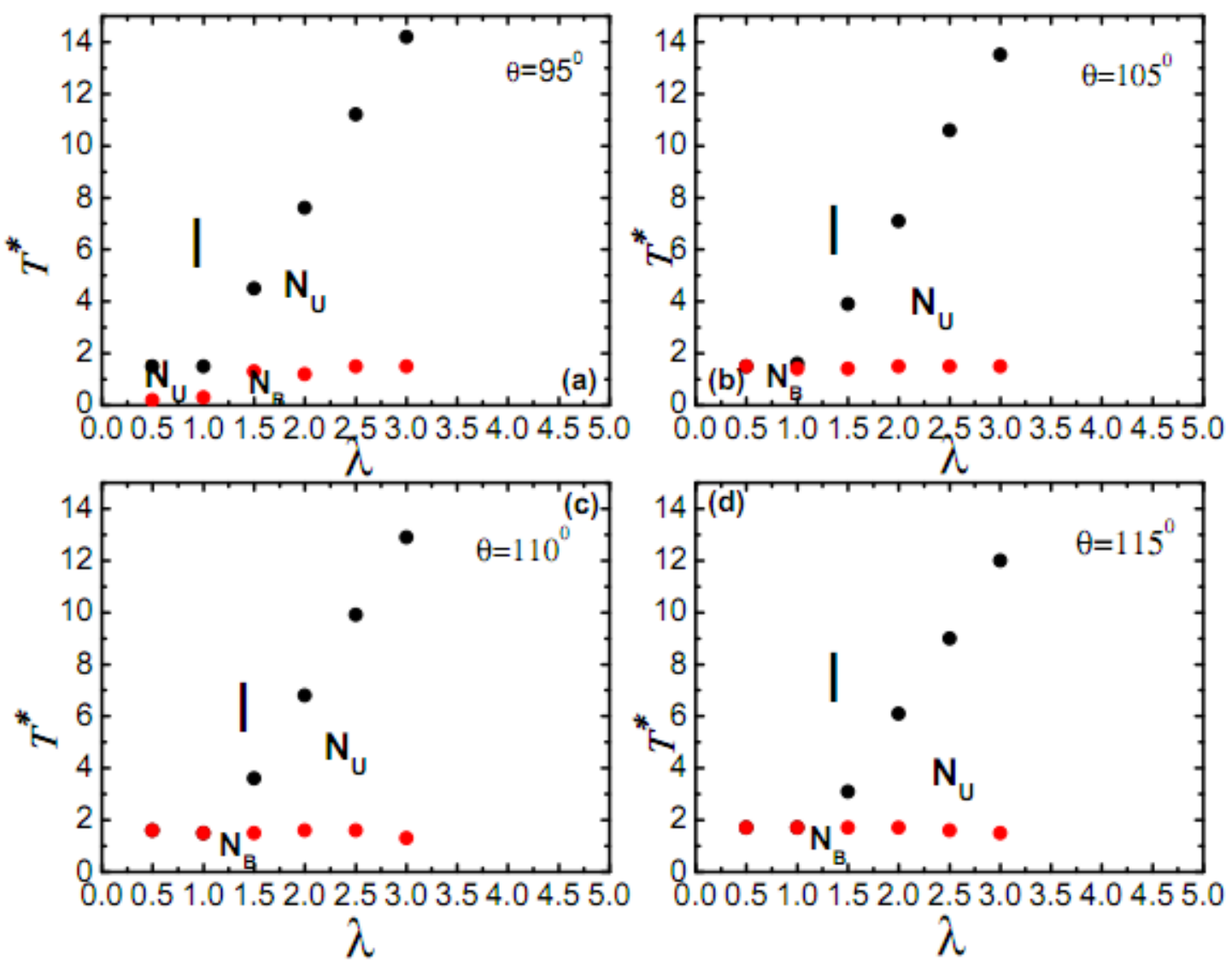

The phase diagrams of liquid crystal formed by bent-core molecules, as a function of $\mathrm{T}^{*}$ and $\lambda$ for four different bent angles. 